\title{
Une hyperéosinophilie révélatrice d'un Syndrome de CHURG et STRAUSS
}

\author{
Djaha Kouassi Jean-Mermoze \\ Gbané Mariam \\ Hounsounou Mariano \\ Michozounou Muriel \\ Coulibaly Abidou Kawélé \\ Diomandé Mohamed \\ Ouattara Baly \\ Eti Edmond
}

Service de Rhumatologie CHU de Cocody, Abidjan-Côte-d' Ivoire

Doi:10.19044/esj.2021.v17n14p140

Submitted: 21 December 2020

Accepted: 05 April 2021

Published: 30 April 2021
Copyright 2021 Author(s)

Under Creative Commons BY-NC-ND

4.0 OPEN ACCESS

Cite As:

Jean-Mermoze D.K., Mariam G., Mariano H., Kawélé C.A., Mohamed D., Baly O. \& Edmond E. (2021). Une hyperéosinophilie révélatrice d'un Syndrome de CHURG et STRAUSS. European Scientific Journal, ESJ, 17(14), 140. https://doi.org/10.19044/esj.2021.v17n14p140

\section{Resume}

Le syndrome de Churg et Strauss est une vascularite nécrosante et granulomateuse systémique des petits et moyens vaisseaux. Nous rapportons un cas de syndrome de Churg et Strauss révélé par un asthme fébrile, une polyarthralgie et une hyperéosinophilie dans un contexte d'altération de l'état général. La recherche d'ANCA au bilan immunologique était négative. La biopsie cutanée n'a pas montré de granulome extravasculaire. L'évolution était favorable sous traitement.

Mots-clés : Polyarthralgie, asthme, hyperéosinophilie, Churg-Strauss 


\title{
Hypereosinophilia Indicative of CHURG and STRAUSS Syndrome
}

\author{
Djaha Kouassi Jean-Mermoze \\ Gbané Mariam \\ Hounsounou Mariano \\ Michozounou Muriel \\ Coulibaly Abidou Kawélé \\ Diomandé Mohamed \\ Ouattara Baly \\ Eti Edmond
}

Service de Rhumatologie CHU de Cocody, Abidjan-Côte-d'Ivoire

\begin{abstract}
Churg-Strauss syndrome is a systemic necrotizing and granulomatous vasculitis of the small and medium vessels. We report a case of Churg-Strauss syndrome revealed by febrile asthma, polyarthralgia and eosinophilia in a context of deterioration of general condition. The ANCA research for immunologic balance sheets was negative. Skin biopsy did not show extravascular granuloma. The evolution was favorable under treatment.
\end{abstract}

Keywords: Polyarthralgia, asthma, hypereosinophilia, Churg-Strauss

\section{Introduction}

Le syndrome de Churg et Strauss (SCS) est une vascularite systémique décrite pour la première fois en 1951 par Jacob Churg et Lotte Strauss comme un syndrome associant un asthme sévère, une fièvre, une hyperéosinophilie, une neuropathie périphérique et des signes d'atteintes cardiaques et rénales (Churg \& al, 1951). Le système nerveux central est plus rarement touché (Noth $\&$ al, 2003; Guillevin \& al, 1999). Nous rapportons un cas de syndrome de Churg Strauss chez une patiente de 31 ans immunocompétente hospitalisée dans le service de rhumatologie du CHU de Cocody à Abidjan en Côte d'Ivoire.

\section{Observation}

Une patiente de 31 ans immunocompétente hospitalisée dans le service de rhumatologie pour une polyarthralgie inflammatoire bilatérale, asymétrique, migratrice. Cette polyarthralgie évoluait depuis 2 mois par poussée et rémission intéressant les chevilles, les genoux, les épaules, le coude gauche et les mains avec des acroparesthésies des mains et des pieds sans douleurs musculaires associés. Le tableau clinique évoluait dans un 
contexte de fièvre intermittent avec une altération de l'état général. La patiente avait aussi un asthme de découverte récente depuis 11 mois associé à un purpura et une urticaire sur le tronc et les membres, de nodules apparus sur le cuir chevelu depuis 2 mois. Elle n'avait pas de douleur abdominale, ni de nausées, ni de vomissements, ni de diarrhée, ni de toux ni de douleur thoracique ni de notion d'allergie répétée ni de terrain atopique.

L'examen physique a objectivé une fièvre à $38,7^{\circ} \mathrm{c}$; une douleur cotée à $7 / 10$ sur l'échelle visuelle analogique ; un indice articulaire à 09 , une amyotrophie des éminences thénar et hypothénar et des interosseux plus marquée à gauche sans déformations articulaires associées, un déficit du fléchisseur commun des doigts à gauche (figure 1); une hypoesthésie cutanée des pieds plus marquée à gauche.

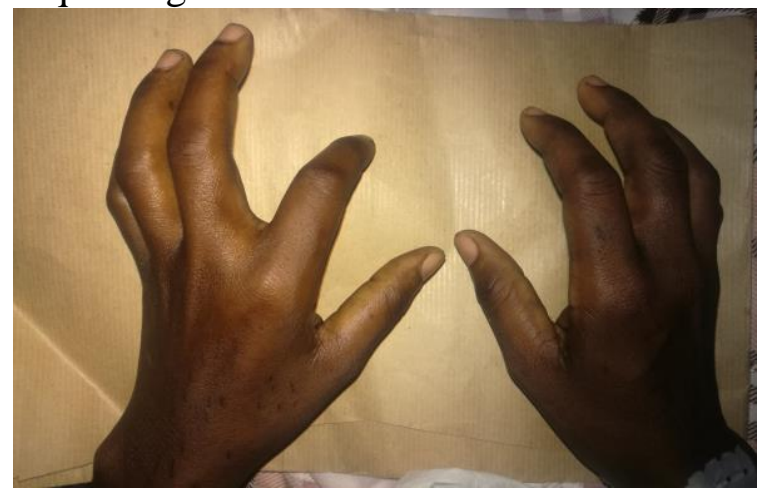

Figure 1 : Déficit du fléchisseur commun des doigts droit et gauche

A l'examen on notait aussi des lésions cutanées purpuriques à la face antérointerne de la cuisse gauche (figure 2).

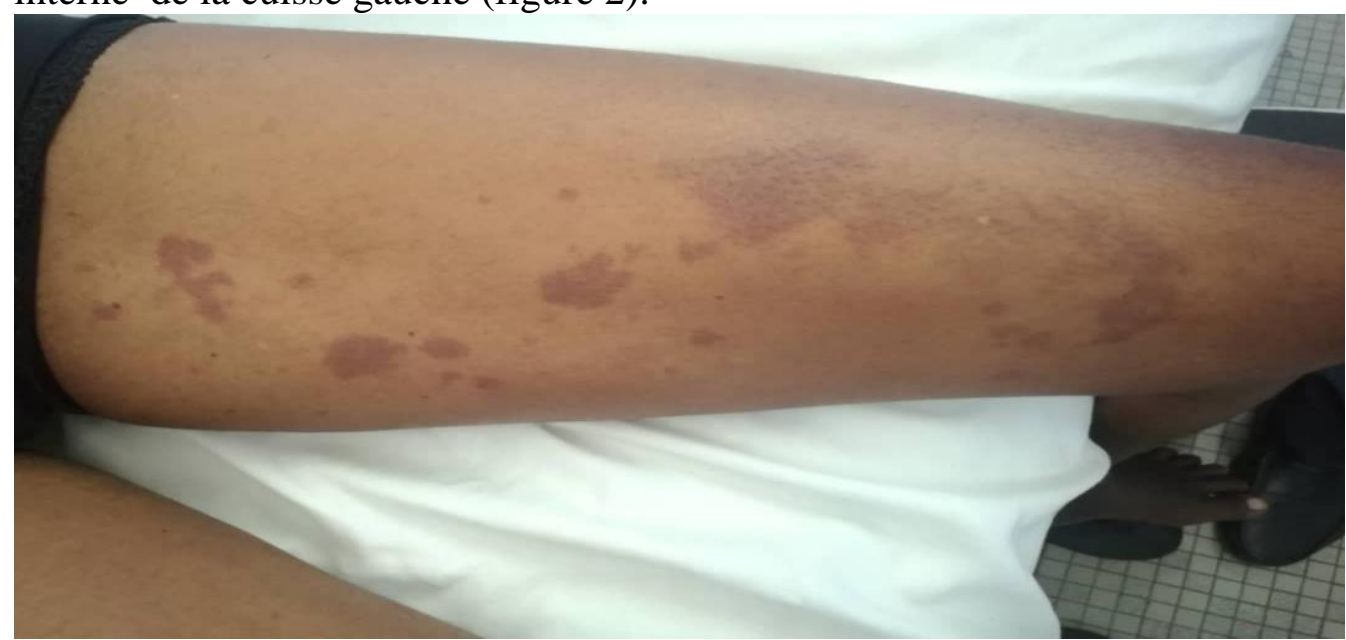

Figure 2 : Lésion cutanées purpuriques de la cuisse gauche 
La biologie montrait : un syndrome inflammatoire (CRP à $48 \mathrm{mg} / \mathrm{L}$, VS à $115 \mathrm{~mm}$ ), une anémie normochrome normocytaire à $9,1 \mathrm{~g} / \mathrm{dl}$, globules blancs $=13650 / \mathrm{mm}^{3}$, une hyperéosinophilie à $7520 / \mathrm{mm}^{3}$, l'urée $=0,20 \mathrm{~g} / 1$, la créatinine $=9 \mathrm{mg} / 1$, protéinurie des $24 \mathrm{~h}=80 \mathrm{mg} / 24 \mathrm{~h}$.

La recherche d'ANCA était négative au bilan immunologique.

La biopsie cutanée faite n'a pas montré de granulome extravasculaire. La radiographie pulmonaire notait des infiltrats alvéolaires mal systématisés (figure 3).

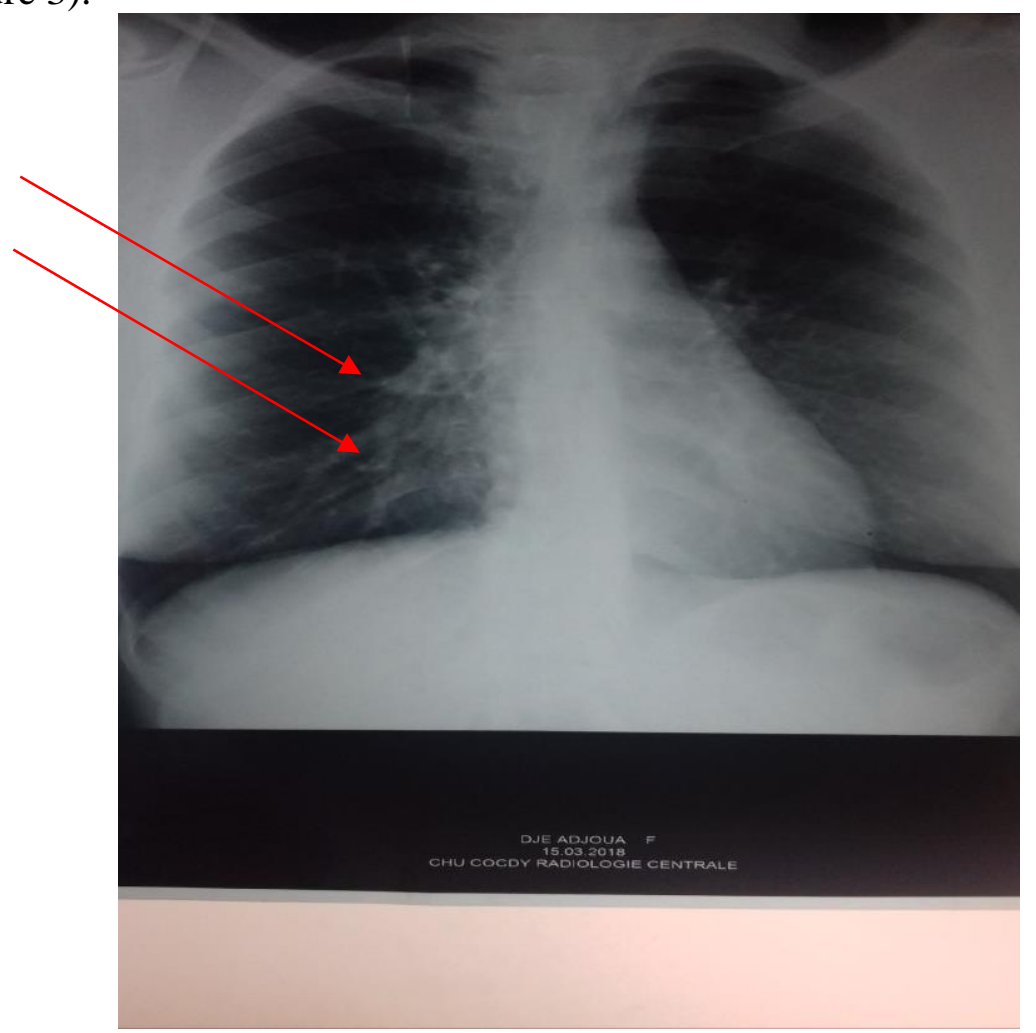

Figure 3 : Radiographie pulmonaire montrant des infiltrats alvéolaires mal systématisés

Les radiographies des mains et des avant-pieds ne notent pas de lésions ostéo-articulaires.

Le diagnostic de Syndrome CHURG et STRAUSS a été retenu devant l'asthme tardif, la fièvre, la polyarthralgie, l'altération de l'état général, le purpura et l'hyperéosinophilie à $7520 / \mathrm{mm}^{3}$.

Elle a été mise sous traitement fait de corticothérapie et d'immunosuppresseurs (cyclophosphamide) et d'une rééducation des mains.

L'évolution sous ce traitement a été très favorable avec régression des symptômes après un recul d'un an. 


\section{Discussion}

Le syndrome de Churg et Strauss est une affection rare et est parmi les moins fréquentes des vascularites systémiques (Lhote \& al, 2007). Il peut toucher tous les âges avec une fréquence maximale entre 30 et 50 ans (Lhote $\&$ al, 2007) comme chez notre patiente qui avait 31 ans. Les arthralgies sont les plus fréquentes des manifestations articulaires et associées aux myalgies participent au syndrome algique.

Toutes les articulations peuvent être atteintes et les arthralgies sont souvent migratrices (Lhote $\&$ al, 2007) tel que observée dans notre cas avec une polyarthralgie migratrice. Notre patiente avait un asthme tardif comme le rapporte la littérature (Solans \& al, 2001 ; Sable \& al, 2005 ; Sinico \& al, 2005) avec un asthme présent chez $100 \%$ des patients ayant un syndrome de Churg et Strauss. Les lésions cutanées à type de purpura ont été retrouvées chez notre patiente. Une atteinte cutanée est retrouvée chez 40 à $75 \%$ des patients (Guillevin \& al, 2012). Il s'agit d'un purpura palpable, souvent nécrotique ayant du corps (Guillevin \& al, 2012). Le purpura était présent dans les études de Haas \& al, 1991 et de Reid \& al, 1998 à des proportions respectives de 25 $\%$ et $26 \%$. Des signes neurologiques présents étaient : une amyotrophie des éminences thénar et hypothénar et des interosseux plus marquée à gauche, un déficit du fléchisseur commun des doigts à gauche, de l'extenseur de l'avantbras gauche et une hypoesthésie cutanée des pieds plus marquée à gauche. Les atteintes neurologiques sont fréquentes au cours du SCS selon la littérature (Della \& al, 2002 ; Keogh \& al, 2003 ; Sable \& al, 2005) et sont le plus souvent des neuropathies périphériques. L'atteinte du système nerveux central est rare et est un facteur de mauvais pronostic (Guillevin \& al, 1996). Une hyperéosinophilie à $7520 / \mathrm{mm}^{3}$ a été retrouvée à la biologie. L'association d'un asthme, d'une fièvre, d'une altération de l'état général et d'une hyperéosinophilie $>1500 / \mathrm{mm}^{3}$ permet de faire le diagnostic de syndrome de Churg Strauss (Guillevin \& al, 2012). La recherche d'ANCA était négative chez notre patiente.

Les ANCA sont présent dans 35 à $40 \%$ des cas dans le Syndrome de Churg et Strauss (Sable \& al, 2005 ; Sinico \& al, 2005). L'évolution de notre patiente était bonne sous corticothérapie et immunosuppresseur (cyclophosphamide) après un recul d'un an. Le syndrome de Churg-Strauss fait appel à l'administration de glucocorticoïdes dont l'activité antiéosinophilique est remarquable et d'un immunosuppresseur principalement le cyclophosphamide ou l'azathioprine dans les formes graves (Cogan \& al, 2007).

\section{Conclusion}

Devant un asthme fébrile associé à une polyarthralgie et à une hyperéosinophilie supérieure à $1500 / \mathrm{ml}$ dans un contexte d'altération de l'état 
général, il faut penser à une vascularite type CHURG et STRAUSS. La recherche d'ANCA au bilan immunologique peut être négative. La biopsie cutanée peut être non contributive. Devant toute hyperéosinophilie, il ne faut pas occulter le syndrome de CHURG et STRAUSS dans la recherche étiologique. Le SCS est rare, il est le premier cas que nous avons diagnostiqué dans le service de rhumatologie. La prise en charge est faite par les corticoïdes et les immunosuppresseurs.

\section{References :}

1. Churg J. \& Strauss L. (1951). Allergic granulomatosis, allergic angiitis and periarteritisnodosa. Am J Pathol ; 27 : 277-330.

2. Noth I., Strek ME. \& Leff AR. (2003). Churg-Strauss Syndrome. Lancet ; $361: 587-94$.

3. Guillevin L., Cohen P., Gayraud M., Lhote F., Jarousse B. \& Casassus P. (1999). Churg-Strauss syndrome. Clinical study and long-term follow-up of 96 patients. Medicine ; $78: 26-37$.

4. Lhote F. (2007). Syndrome de Churg et Strauss. Presse Med ; $36: 875$ 89.

5. Solans R., Bosch JA., Perez-Bocanegra C., Selva A., Huguet P., Alijotas J. \& al. (2001). Churg-Strauss syndrome : outcome and longterm follow-up of 32 patients. Rheumatol ; 40 : 763-71.

6. Sablé-Fourtassou R., Cohen P., Mahr A., Pagnoux C., Mouthon L., Jayne D. \& al. (2005). Anti-neutrophil cytoplasmic antibodies and the Churg-Strauss syndrome. Ann Int Med ; 143 : 632-8.

7. Sinico RA., Di Toma L., Maggiore U., Bottero P., Radice A., Tosoni C. \& al. (2005). Prevalence and clinical significance of antineutrophil cytoplasmic antibodies in Churg-Strauss syndrome. Arthritis Rheum. ; $52: 2926-35$.

8. Guillevin. L. (2012). La granulomatose éosinophilique avec polyangéite (Syndrome de Churg et Strauss). Presse med ; 41 : 100413.

9. Hass C., Geneau C., Odinot De Jaeger C., Lavner M., Lowenstein W., Choubrac P. \& al. (1991). L'angéite allergique avec granulomatose: Syndrome de Churg et Strauss. Etude rétrauspective de 16 observations. Ann Med Interne (Paris) ; 142 : 335-42.

10. Reid AJ., Harrison BD., Watts RA., Watkin SW., McCann BG. \& Scott DG. (1998). Churg-Strauss in district hospital. QJM ; 91 : 21929.

11. Della Rossa A., Baldini C., Tavoni A., Tognetti A., Neglia D., Sambuceti G. \& al. (2002). Churg-Strauss syndrome : clinical and serological features of 19 patients from a single italian centre. Rheumatology (Oxford) ; 41 : 1286-94. 
12. Keogh KA. \& Specks U. (2003). Churg-Strauss Syndrome : clinical presentation, antineutrophil cytoplasmic antibodies and leukotriene receptor antagonist. Am J Med ; 115 : 284-90.

13. Sable-Fourtassou R., Cohen P., Mahr A., Pagnoux C., Mouthon L., Jayne D. \& al. (2005). Antineutrophil cytoplasmic antibodies and the Churg-strauss syndrome. Ann Intern Med ; 143 (9) : 632-8.

14. Guillevin L., Lhote F., Cacassus P., Cohen P., Jarrousse B. \& Lortholary O. (1996). Prognostic factors in polyarteritis nodosa and Churg-Strauss Syndrom. A prospective study in 342 patients. Medicine ; $75: 17-28$.

15. E. Cogan. \& F. Roufosse. (2007). Eosinophilie, syndrome de ChurgStrauss et Syndrome hyperéosinophilique. Rev Med Int ; 28S : S256S58. 